\title{
Silica passivated conjugated polymer nanoparticles for biological imaging applications
}

\author{
Struan Bourke ${ }^{* a}$, Laura Urbano ${ }^{\mathrm{b}}$, Antoni Olona ${ }^{\mathrm{c}}$, Ferran Valderrama ${ }^{\mathrm{c}}$, Lea Ann Dailey ${ }^{\mathrm{b}}$ and Mark A. \\ Green $^{\mathrm{a}}$ \\ ${ }^{2}$ Department of Physics, King's College London, London, UK, WC2R 2LS; \\ ${ }^{b}$ Department of Pharmacy and Forensic Sciences, King's College London, London, UK, SE1 9NH \\ ${ }^{\circ}$ Cell Biology and Genetics Research Centre, St. George's University of London, London, UK SW17 \\ ORE \\ *struan.bourke@kcl.ac.uk
}

\begin{abstract}
Colorectal and prostate cancers are major causes of cancer-related death, with early detection key to increased survival. However, as symptoms occur during advanced stages and current diagnostic methods have limitations, there is a need for new fluorescent probes that remain bright, are biocompatible and can be targeted. Conjugated polymer nanoparticles have shown great promise in biological imaging due to their unique optical properties. We have synthesised small, bright, photo-stable CN-PPV, nanoparticles encapsulated with poloxamer polymer and a thin silica shell. By incubating the CN-PPV silica shelled cross-linked (SSCL) nanoparticles in mammalian (HeLa) cells; we were able to show that cellular uptake occurred. Uptake was also shown by incubating the nanoparticles in RWPE-1, WPE1-NB26 and WPE1NA22 prostate cancer cell lines. Finally, HEK cells were used to show the particles had limited cytotoxicity.
\end{abstract}

Keywords: Conjugated polymers, nanoparticles, silica shells, biological imaging, cancer imaging

\section{INTRODUCTION}

Advancements in imaging have increased the understanding of how biological processes occur, providing greater understanding of the link between structure and function ${ }^{1}$. By studying how diseases work, and identifying early signs and symptoms, it is possible to better treat the condition as well as determine which therapies are most effective.

After cardiovascular disease, cancer is considered one of the leading cause of death worldwide with 8.2 million cancer related deaths in $2012^{2,3}$. This global burden is increasing due to a rapidly growing population, as well as people living longer leading to an aged population. Factors such as genetics and lifestyle also lead to an increase rates of cancer ${ }^{4}$. Cancer is a broad term for a diverse group of diseases that affect all tissues within the body, and is understood to be the uncontrolled proliferation of cells leading to metastases. In the UK, prostate and colorectal cancer are the second and fourth most common cancers and count for $25 \%$ of all new cases ${ }^{5,6}$. Diagnosis for both tend to occur late, leading to a high fatality rate: bowel cancer accounts for $10 \%$ of all cancer deaths in the UK and prostate cancer accounts for $13 \%$ of all cancer deaths in men ${ }^{5,6}$. While the ultimate goal would be to cure cancer in all its forms, there is a need to develop a more efficient and rapid method for diagnosing cancer in the early stages. Several non-invasive imaging methods are currently used in the diagnosis, including computed tomography (CT), magnetic resonance (MRI), positron emission tomography (PET), and single photon emission CT (SPECT) ${ }^{7}$. However, there is no one ideal technique that can be substituted for the others, and most diagnoses are obtained from a combination of several different imaging techniques ${ }^{8}$.

Current optical imaging techniques use organic fluorescence dyes as probes. These dyes have some drawbacks such as unsatisfactory brightness, rapid photo bleaching and small Stokes' shifts, which cause difficulty in detecting the signal ${ }^{9}$. Therefore materials that are bright, have greater photo-, bio-, and environmental stability, and a large Stokes shift are now being developed for fluorescence microscopy and clinical imaging ${ }^{10}$.

Reporters, Markers, Dyes, Nanoparticles, and Molecular Probes for Biomedical Applications IX edited by Samuel Achilefu, Ramesh Raghavachari, Proc. of SPIE Vol. 10079, 100790A

(C) 2017 SPIE · CCC code: 1605-7422/17/\$18 · doi: 10.1117/12.2252035 
In recent years there has been a focus on the development of quantum dots (QDs) which are inorganic semiconductors with tuneable emission. They have bright fluorescence, excellent photo-stability and narrow emission ${ }^{11}$. However, as they are normally composed of heavy metals, such as cadmium and selenium, there is a serious concern about the toxicity of the nanoparticles to cells ${ }^{12,13,14}$. There is another set of fluorescent particles that have been studied, composed of conjugated polymers (CPs) which have gained growing attention due to their attractive optical properties such as bright photoluminescence and photo-stability ${ }^{15-19}$. CPs are organic semiconductors with backbone of alternating multiple and single bonds which exhibit efficient coupling between optoelectronic segments; electrons can delocalise allowing for excitons to diffuse efficiently throughout the polymer chain ${ }^{20,21}$. There are a number of advantages of conjugated polymer nanoparticles (CPNs) over QDs, including their ease of processing, large absorption coefficients, tuneable optical properties, controllable dimensions and biologically inert components circumventing the issue of heavy metal toxicity of QDs ${ }^{19,22}$. It is also possible to obtain conjugated polymers commercially in a range of colours that allows the potential for tuneable emission.

Despite the advantages of CPNs, the major limitation is their hydrophobic characteristics. For example, the majority of conjugated polymers need to be dissolved in solvents with a surfactant to make CPNs water stable. The nanoparticles are synthesised using two methods: mini-emulsion and nanoprecipitation. The mini-emulsion method involves dissolving the $\mathrm{CP}$ in an aprotic, water immiscible solvent then emulsifying the solution through sheer force in an aqueous solution that contains surfactant ${ }^{23-26}$. In contrast, the nano-precipitation method involves dissolving the $\mathrm{CP}$ into a water-miscible solvent such as THF and then injecting it into a non-solvent (such as water). The mixture is stirred vigorously, normally by sonication, and the organic solvent is removed via evaporation leaving behind the dispersed nanoparticles ${ }^{15,27}$. With both methods, the main driving force for the formation of nanoparticles is the hydrophobic effect ${ }^{22}$ : the polymer chains fold in on themselves to avoid exposure to water. Competition between aggregation and collapse of the polymer chains means the size of the nanoparticles can be controlled ${ }^{28}$, normally between 5-30 nm. This depends on factors such as polymer concentration, solubility, water temperature and strength of sonification ${ }^{25}$.

We have developed a number of ways to encapsulate CPs inside micelles of different chemical compositions, such as hydrophobins, and these are then imaged in HeLa cells ${ }^{29}$. We have also studied the effect of surface chemistry of CPNs and how this affects uptake and internalisation in phagocytic cells ${ }^{30}$. We report the possibility of using silica to encapsulate a CP/pluronic micelle which is prepared using a modified bioinspired silification approach ${ }^{31,32}$. The process involves simultaneously encapsulating a conjugated polymer inside the micelles, then forming a silica layer between the interface of the core and shell. The optical properties of the CP loaded silica shelled cross-linked (SSCL) micelles are shown using a variety of cancer cell lines (HeLa, RWPE-1, WPE1-NB26 and WPE1-NA22) with cytotoxicity determined using HEK cells. The ability of these nanoparticles as fluorescent probes is shown via the uptake of the particles by the different cells and subsequent imaging with a confocal scanning laser microscope.

\section{METHODOLOGY}

\subsection{Reagents and Materials}

Poly(2,5-di(hexyloxy)cyanoterephthalylidene) (CN-PPV) (MW=Unknown), pluronic F127 (MW=12.5 kDa avg), tetrahydrofuran (with $250 \mathrm{ppm}$ BHT as inhibitor, 99.9\%) (THF) and tetramethyl orthosilicate (99\%) (TMOS) were purchased from Sigma-Aldrich (England, UK). Dulbecco's Modified Eagle Medium (DMEM), fetal bovine serums (FBS), Keratinocyte-Serum Free Medium (SFM), bovine pituitary extract (BPE), human recombinant epidermal growth factor (EGF) were purchased from Thermofisher Scientific (England, UK). CellTiter-Glo ${ }^{\circledR}$ reagent was purchased from Promega (England, UK).

HeLa cells were supplied by Sergi Garcia-Maynes' Lab in the Department of Physics at King's College London. HEK cells were supplied by Maryna Panamarova from the Zammit Group in the Randall Division of Cellular and Molecular Biophysics, King's College London. RWPE-1, WPE1-NB26 and WPE1-NA22 were supplied by Ferran Valderamma's $\mathrm{Lab}$ at the University of St. George's, London.

\subsection{Instrumentation}

Absorption spectra were measured using a Hitachi U-4100 UV-Visible-NIR spectrometer using a $1 \mathrm{~cm}$ path length quartz cuvette. Photoluminescence spectra were measured using a Horiba Fluoromax-4 spectrofluorometer. Particle size 
distributions were obtained using both a Malvern Nanosight (utilising nanoparticle tracking analysis) and a Malvern Zetasizer (utilising dynamic light scattering). Zeta potential was obtained on a Malvern Zetasizer. Transmission electron microscopy images were acquired on a Hitachi 7100 at St George's University of London, with a filament electron source at $100 \mathrm{kV}$. Image analysis was performed with ImageJ software. Quantum yields were acquired on a Hamamatsu Quantaurus-QY Absolute PL quantum yield spectrometer, with samples excited at $450 \mathrm{~nm}$ with a bandwidth of 5-7 $\mathrm{nm}$. Cells were imaged on an A1 inverted confocal at the Nikon Centre at King's College London.

\subsection{Synthesis of conjugated polymers loaded into silica-shell cross-linked polymeric micelles (CP-SSCL)}

The bioinspired silification method was adapted from work by ${ }^{31-33}$. CN-PPV was prepared in THF by dissolving $1 \mathrm{mg}$ of polymer into $1 \mathrm{ml}$ of THF to make a $1 \mathrm{mg} / \mathrm{ml}$ polymer solution. The solution was sonicated in a $35 \mathrm{kHz}$ ultrasound bath at $7-9{ }^{\circ} \mathrm{C}$, in 30 second bursts for 5 minutes to ensure the polymer was completely dissolved. In a separate flask, $2 \mathrm{mg}$ of the pluronic F127 was added to $1 \mathrm{ml}$ of THF $(2 \mathrm{mg} / \mathrm{ml}$ stock) and sonicated for 5 minutes. $100 \mu \mathrm{L}$ of the CN-PPV polymer solution was added to the $\mathrm{F} 127$ solution and left stirring overnight at $45^{\circ} \mathrm{C}$. Upon cooling to room temperature, $100 \mu \mathrm{l}$ of TMOS was added. The solution was then injected into $13.5 \mathrm{ml}$ of ice-cold deionised water and sonicated for 10 minutes. The solution was then stirred continuously at $400 \mathrm{rpm}$, at room temperature, for four days to evaporate off THF and to ensure complete hydrolysis of TMOS at the interface between the core and corona of the F127 micelles. Loss of water was compensated by readjustment to $10 \mathrm{ml}$. The nano-suspension $(10 \mu \mathrm{m} / \mathrm{ml}$ of CN-PPV or total solid of 210 $\mu \mathrm{m} / \mathrm{ml}$ ) was subsequently filtered through a $0.2 \mu \mathrm{m}$ cellulose acetate Gilson syringe filter. The filtrate was stored at room temperature.

\subsection{CP-SSCL associated with cells}

HeLa and HEK cells were cultured as separate adherent monolayers DMEM supplemented with $10 \%$ heat inactivated FBS. HeLa cells were cultured on a sterilised 8 square well microplate, and HEK cells were cultured on a sterilised 96 well microplate. Cell cultures were kept at physiological temperature $\sim 37^{\circ} \mathrm{C}, 5 \% \mathrm{CO}_{2}$ in a humidified incubator. The CN-PPV nanoparticle suspension was serially diluted in DMEM to have a range of polymer concentrations $(7.5 \mu \mathrm{m} / \mathrm{ml}$ to $0.1 \mu \mathrm{m} / \mathrm{ml}$ ). $100 \mu \mathrm{l}$ of the CN-PPV nanoparticle suspension was added to $200 \mu \mathrm{L}$ of the aforementioned media (for the 8 well plate) and $20 \mu \mathrm{l}$ of the CN-PPV nanoparticle suspension was added to $100 \mu \mathrm{l}$ of the aforementioned media (for the 96 well plate). These were incubated for 24 hours. RWPE-1, WPE1-NB26 and WPE1-NA22 cells were cultured as adherent monolayers in SFM supplemented with $0.05 \mathrm{mg} / \mathrm{ml} \mathrm{BPE}$ and $5 \mathrm{ng} / \mathrm{ml}$ human recombinant EGF, on a sterilised 24 well plate. Cell cultures were kept at physiological temperature $\sim 37^{\circ} \mathrm{C}, 5 \% \mathrm{CO}_{2}$ in a humidified incubator. The $\mathrm{CN}$ PPV nanoparticle suspension was serially diluted in the SFM to have a range of polymer concentrations $(7.5 \mu \mathrm{m} / \mathrm{ml}$ to $0.1 \mu \mathrm{m} / \mathrm{ml}$ ) and $500 \mu \mathrm{l}$ of the CN-PPV nanoparticle suspension was added to $2 \mathrm{~mL}$ of the aforementioned media and incubated for 24 hours.

HeLa, RWPE-1, WPE1-NB26 and WPE1-NA22 cells were fixed in a 4\% formaldehyde solution for 15 minutes, and then washed with phosphate-buffered saline (pH 7.0) six times. All images were acquired on a Nikon A1 inverted confocal using a $\times 20$ dry objective lens of numerical aperture 0.75 , with $488 \mathrm{~nm}$ excitation. Emission from the CN-PPV nanoparticles was collected in two channels, $570-620 \mathrm{~nm}$ and $663-738 \mathrm{~nm}$ with $2 \times$ line averaging. The brightest emission was observed in the $570-620 \mathrm{~nm}$ channel. Z-stack images were collected every $0.125 \mu \mathrm{m}$, with $5.75 \mu \mathrm{m}$ sample depth. All images are displayed with $0.2 \%$ saturation pixel thresholding.

For the cytotoxicity study with HEK cells, after the time intervals of 1 hour, 24 hours and 48 hours, $100 \mu \mathrm{L}$ CellTiterGlo® reagent was added and cells were left to lyse for 10 minutes before the luminescence was recorded. 


\section{RESULTS AND DISCUSSION}

\subsection{Preparation and physical characterization of CP-SSCL}

By using a modified version of the silification method as described by ${ }^{32}$, it was possible to encapsulate CN-PPV into F127 micelles followed by passivation in silica. This involved dissolving the conjugated polymer, F127 block copolymer, and TMOS in THF. The mixture solution was then injected into water under ultrasonication. This change in the environment caused the F127 block copolymer to encapsulate the CN-PPV as a micelle (with the poly(propylene oxide) (PPO) blocks as the core and poly(ethylene oxide) (PEO) blocks as the shell). As both the TMOS and the conjugated polymer are hydrophobic and insoluble in water, they becomes compartmentalised within the PPO core which caused hydrolysis and condensation to be confined at the regions between the core and the shell of the F127 micelles ${ }^{32}$. This forms a sol-gel transfer, resulting in a thin silica layer forming around the PPO core of the F127 micelles.

For the purpose of cell imaging, the red-emitting conjugated polymer, poly(2,5-di(hexyloxy)cyanoterephthalylidene) (CN-PPV), was chosen which has a emission wavelength of $550 \mathrm{~nm}$ in solvent. The chemical structure of the CN-PPV, the structure of the NP and the resultant aqueous suspensions are shown in Figure 1. As can be seen from the figure, the suspension is transparent which suggests that the nanoparticles are stable over a period of at least 3 months, with aggregation only observed in samples older than 2 months that were left at room temperature. This suggested that the conjugated polymer is completely encapsulated by the F127 micelle, and that the suspension emits a bright colour when exposed to a UV light $(\lambda=365 \mathrm{~nm})$.
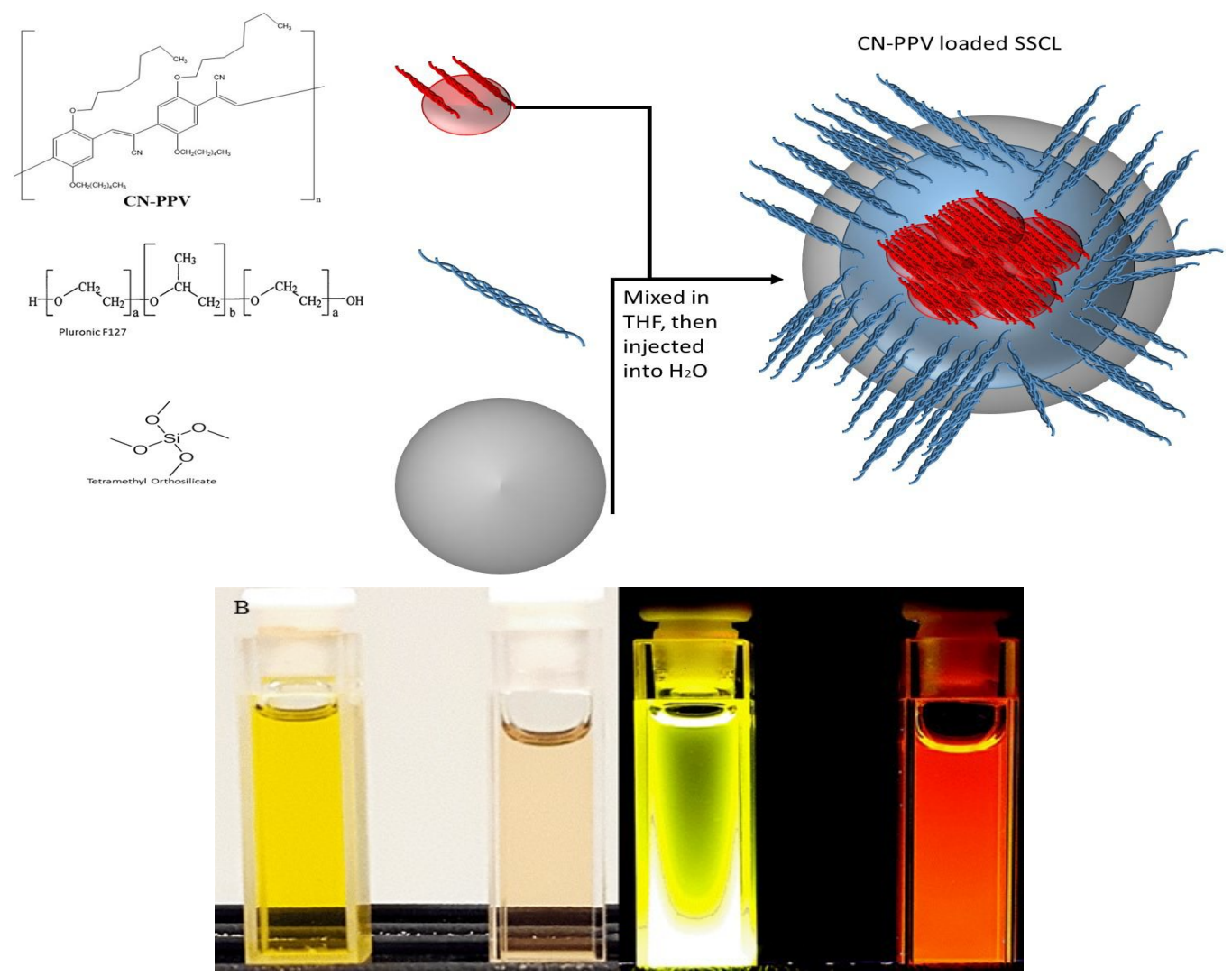

Figure 1: Top, the chemical structures of CN-PPV, F127 AND TMOS and an illustration of the nanoparticle in $\mathrm{H}_{2} \mathrm{O}$. Bottom, CN-PPV in THF (left) and as nanoparticles in aqueous solution (right) in left hand panel. Samples were excited using an UV lamp $(\lambda=365 \mathrm{~nm})$ in right hand panel. 
The size of the particles was determined using dynamic light scattering (DLS). It was observed that the particles had a hydrodynamic diameter of between 40 and $80 \mathrm{~nm}$ (figure 2). The DLS also showed a relatively narrow size distribution, as shown by a low polydispersity index (PDI) of around 0.16 . Transmission electron microscopy (TEM) revealed coreshell structures, as shown in figure 2, with the silica shell appearing darker (due to higher electron density) compared to the PPO blocks of the F127 and the conjugated polymers. The bright cores are likely the F127 containing the CN-PPV. On average, the particle ha a core diameter of $24 \mathrm{~nm}$ and an outer diameter of $30 \mathrm{~nm}$, giving a total diameter of between 50 and $60 \mathrm{~nm}$.
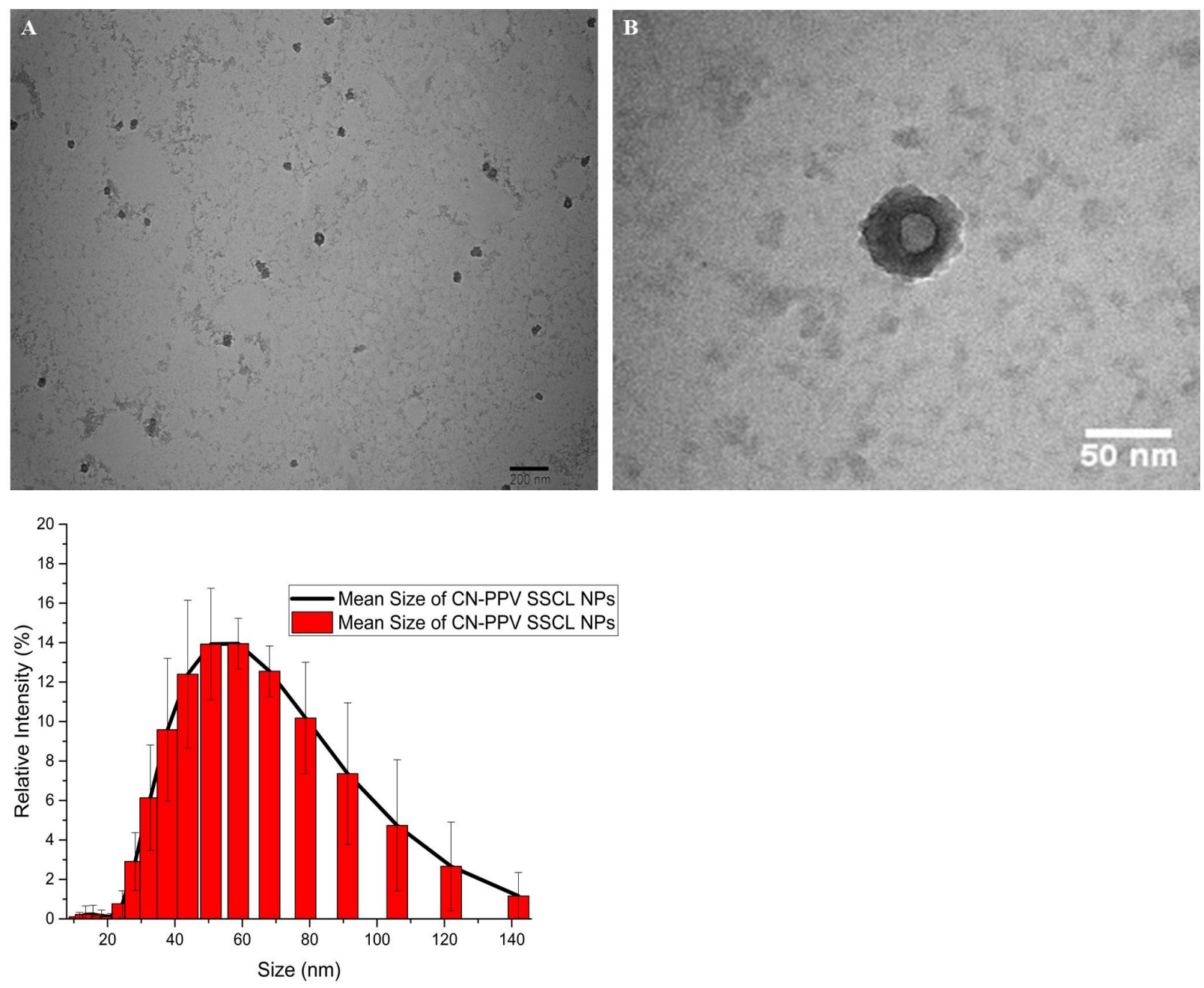

Figure 2: TEM images of the CN-PPV loaded SSCL. A) Taken at 50x resolution (scale bar $=200 \mathrm{~nm}$ ). B) Close up on one nanoparticle at $100 x$ resolution (scale bar $=50 \mathrm{~nm}$ ). The darker outer shell is silica, whereas the lighter inner core is the F127 and CP. C) graph of size vs relative intensity taken from the DLS $(n=4)$.

This relatively uniform size suggests that the micelles containing the conjugated polymer are trapped by the cross-linked silica layer, preventing the micelles from expanding to accommodate the conjugated polymers. The slight difference between the sizes of the particles observed on the TEM vs the DLS measurements is because the DLS measures the hydrated size of the particles in water with their extended free PEO chains ${ }^{32}$. Both F127 micelles containing no conjugated polymer, or conjugated polymer with no silica, were measured to have diameters of $55 \mathrm{~nm}$, which is within the size range observed by ${ }^{34}$. This confirms that the silica layer is forming between the core and the shell of the loaded F127 micelles, allowing the PEO shell to provide aqueous solubility and stabilisation. Table 1 summarises the physical 
characteristics of the CN-PPV SSCLs. The zeta potential was also measured using the Malvern zetasizer in deionised $\mathrm{H}_{2} \mathrm{O}$ at $25^{\circ} \mathrm{C}$. The zeta potential provides indirect information about the nanoparticle surface charge, and the CN-PPV loaded SSCL were found to have a neutral zeta potential value (as shown in table 1). When diluted into DMEM there was no change in the zeta potential.

Table 1: Physical characterisation of the CN-PPV SSCL nanoparticles

\begin{tabular}{|l|c|}
\hline & CN-PPV SSCL \\
\hline Average core diameter $(\mathrm{nm})$ & 24 \\
\hline Average outer diameter $(\mathrm{nm})$ & 30 \\
\hline Hydrodynamic diameter $(\mathrm{nm})$ & $54.53 \pm 3.4$ \\
\hline Polydispersity Index $(\mathrm{PDI})$ & $0.161 \pm 0.015$ \\
\hline Zeta Potential $(\mathrm{mV})$ & -12 \\
\hline
\end{tabular}

\subsection{Optical properties of CP-SSCL}

The optical properties of conjugated polymers are dependent on; a) the initial concentration of polymer, b) the solvent, and c) physical conformation of the chains. It is known that during encapsulation, the conjugated polymer is forced to coil and twist, shortening its effective conjugation length. This increases the number of defects, as well as forming densely packed micelles, which explains the broadening of the absorption spectrum of the particle compared to the $\mathrm{CN}$ PPV in THF. Due to this forced coiling, the polymers interact closely with each other causing delocalisation of the $\pi$ electrons leading to an increased inter-chain aggregate state $^{35}$. While the absorption band broadens due to this, the photoluminescence (PL) spectrum is also affected: a significant red shift occurs, which is likely due to the increased inter-chain interactions. This allows rapid energy transfer from high energy to lower energy segments due to the short conjugated lengths and weakly emissive aggregates. In addition, numerous groups have reported similar observations based on the photo-physical behaviour of water-dispersed conjugated polymer nanoparticles ${ }^{11,15,36}$. The normalised spectra of absorption and photoluminescence are shown in figure 3 (particles excited at $450 \mathrm{~nm}$ ).

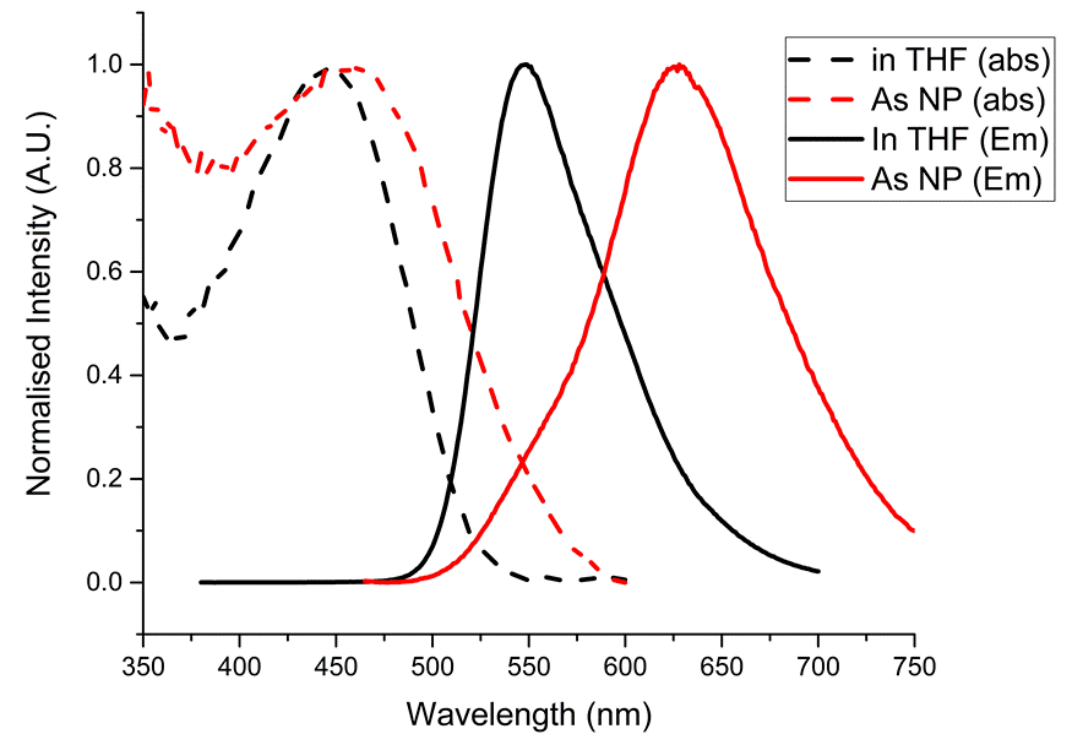

Figure 3: Normalised absorption spectra of CN-PPV in THF (dotted black line), of the nanoparticle (dotted red line) and the photoluminescence spectra of CN-PPV in THF (black line) and as a nanoparticle (red line). $\lambda_{\text {em }}$ for CN-PPV in THF was 550 $\mathrm{nm}$, whereas $\lambda_{\mathrm{em}}$ for the nanoparticles was $623 \mathrm{~nm}$. Sample concentrations were at $10 \mu \mathrm{m} / \mathrm{ml}$. Excitation was at $450 \mathrm{~nm}$. 
The nanoparticle photo-stability over a period of 1 month was also studied: $3 \mathrm{~mL}$ of the aqueous suspension was stored in a quartz cuvette with a sealed lid, and the sample was excited at $450 \mathrm{~nm}$ periodically over a 35 day period, with 3 readings taken at each chosen day. From figure 4 it is shown that while there was a very small red shift (likely due to aggregation of sample), the actual intensity of the sample did not vary significantly over the time period (dropping to $95 \%$ of the initial $(\mathrm{t}=0)$ intensity). Quantum yields were approximately $30 \%$ when excited at $480 \mathrm{~nm}$ using an integrating sphere. The decreased quantum yield compared to $52 \%$ for the polymer in $\mathrm{THF}^{37}$. The decreased emission of the CPNs relative to the polymer form is due to defects in the polymers entrapped within the micelles. The optical properties are summarised in table 2 .
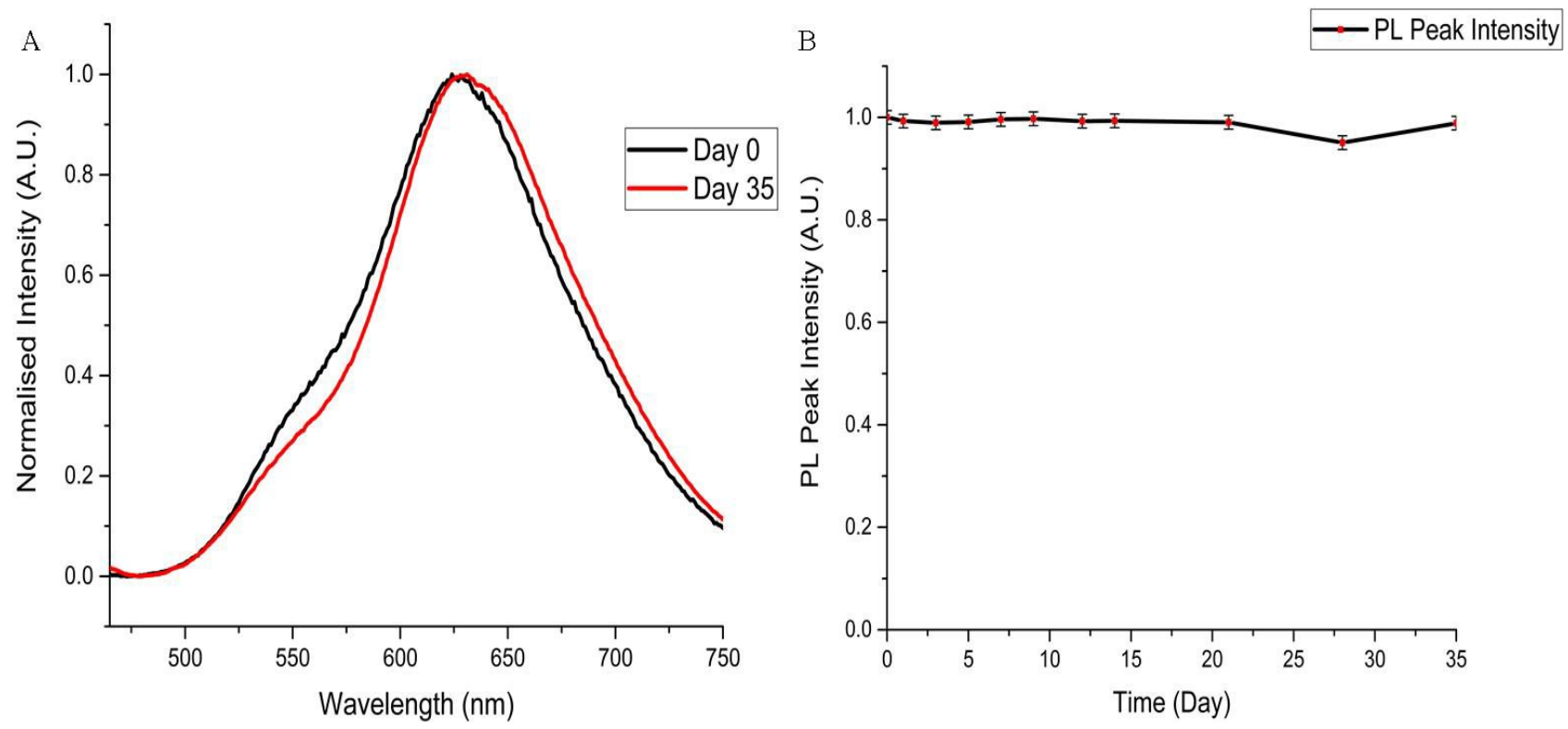

Figure 4: A) normalised photoluminescence peak of sample at day 0 (black) vs Day 35 (red) $\left(\lambda_{\text {em }}=623\right)$. B) The PL peak intensity at different day intervals over the 35 day period. Error bars are standard deviation of the mean. All samples were excited at $450 \mathrm{~nm}$.

Table 2: Optical Properties of CN-PPV SSCL

\begin{tabular}{|l|c|c|}
\hline & CN-PPV in THF & CN-PPV SSCL \\
\hline Absorption peak (nm) & 447 & 458 \\
\hline Emission peak (nm) & 549 & 628 \\
\hline Quantum Yields (QY) (\%) & $52^{*}$ & 30 \\
\hline
\end{tabular}

*Taken as a reference point from ${ }^{3}$

\subsection{In vitro cellular uptake and imaging studies}

The biocompatibility of any new particle used in imaging is important (especially for cellular imaging). In this study, the cytotoxicity of the CN-PPV SSCLs were evaluated by measuring the in vitro viability of human embryonic kidney cells 293 (HEK293) using CellTiter-Glo® Luminescent Cell Viability Assay (Promega, UK) which determined the number of viable cells in culture based on quantitation of the ATP present, to give an indication of metabolically active cells. Figure 5 shows normalised luminescence after 1 hour, 24 hours and 48 hours of exposure to CPNs. These were normalised against the control containing no particles, and showed that CN-PPV loaded SSCL have low cytotoxicity at low concentrations. However, at higher concentrations of CN-PPV loaded SSCL there was a decrease in 
the luminescence in the wells, indicating a decrease in the number of cells. This was particularly the case with the 10 $\mu \mathrm{g} / \mathrm{ml}$ samples, where after 1 hour; all cells appear to have died. It is possible that at higher concentration, the nanoparticles aggregate in the DMEM and affect the cellular environment. The bare CN-PPV particles, without pluronic or Silica are persistently stable and did not seem to affect cell viability.

To investigate their potential use in biological imaging, the CN-PPV loaded SSCL were initially incubated with HeLa cells at a low concentration $(1 \mu \mathrm{g} / \mathrm{ml}$ polymer concentration) for 24 hours. After incubation, the cells were washed with PBS before being fixed in 10\% formalin. The cellular uptake of the CN-PPV loaded SSCL by HeLa cells were evaluated by an A1 inverted confocal microscope. From Figure 6 it can be seen that the CN-PPV loaded SSCL (red) are either located on the surface of the HeLa cells or has been up-taken by the cells.

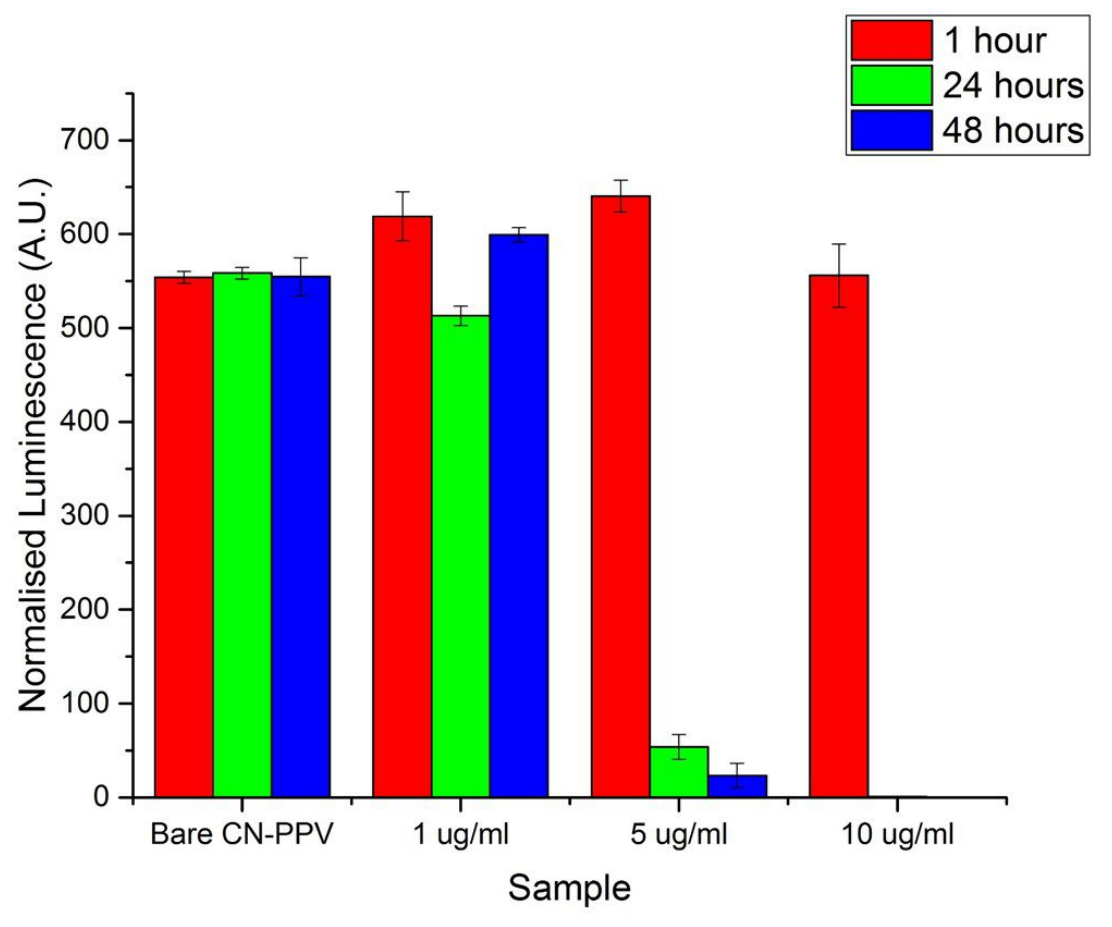

Figure 5: Normalised luminescence of CN-PPV (no surfactant) vs different concentrations of CN-PPV loaded SSCL incubated in HEK cells. These were normalised against control wells with no nanoparticles. 


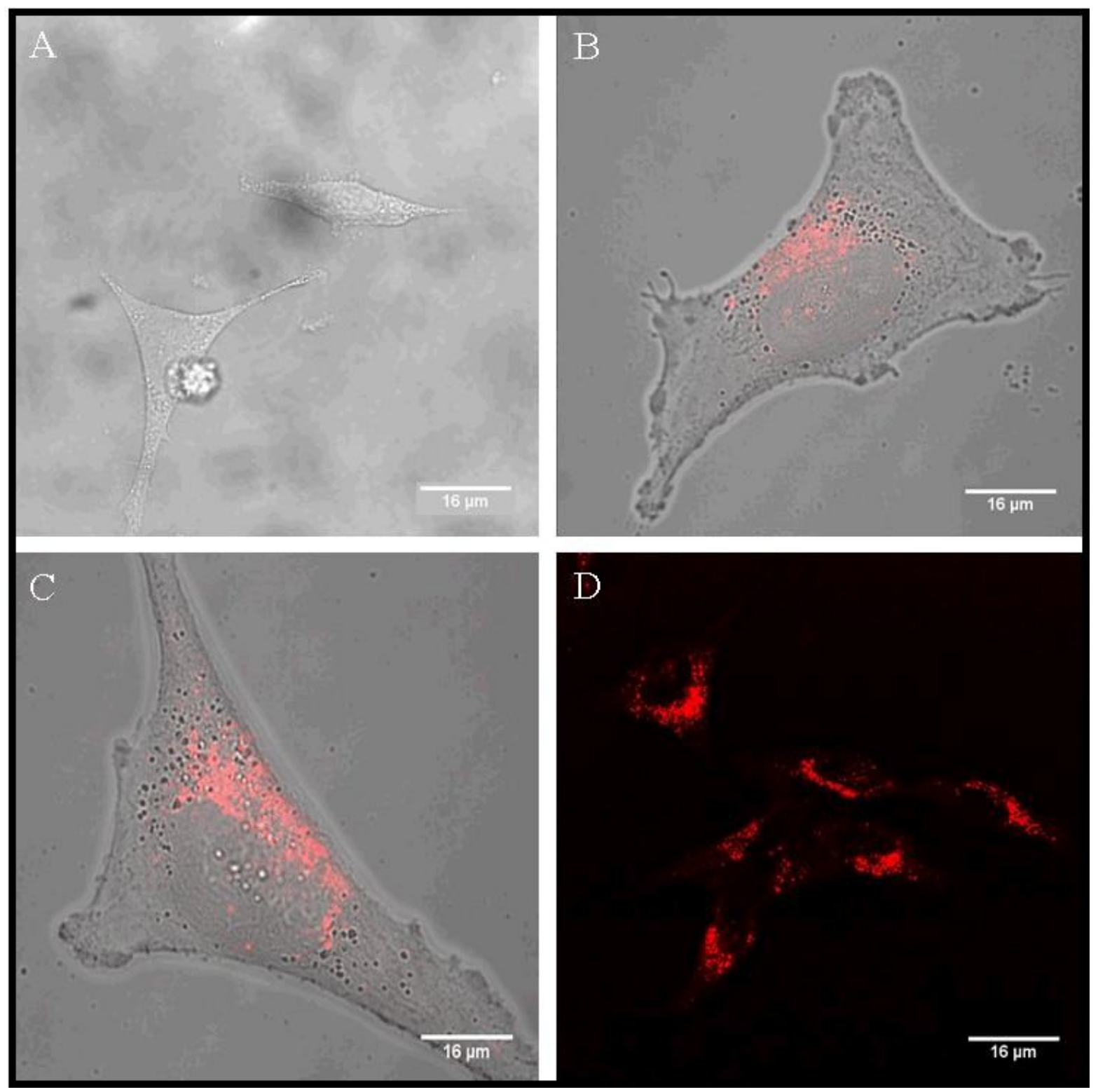

Figure 6: HeLa cells untreated or treated with CN-PPV loaded SSCL. A) untreated HeLa cells at 60x magnification. B) and C) are treated HeLa cells overlayed over transmission light images of the cells. D) HeLa cells treated with $1 \mu \mathrm{g} / \mathrm{ml}$ of nanoparticles at 60x magnification. Fluorescence was detected in the 570-620 $\mathrm{nm}$ channel. Scale bars $=16 \mu \mathrm{m})$.

A Z-stack image was used to visualize whether the CN-PPV loaded SSCL CPNs were internalized by HeLa cells. ZStack images were collected with 37 slices covering a sample depth of $\sim 5.75 \mu \mathrm{m}$, indicating that CPNs were localised inside live HeLa cells, (Figure 7) after 24 hours. 


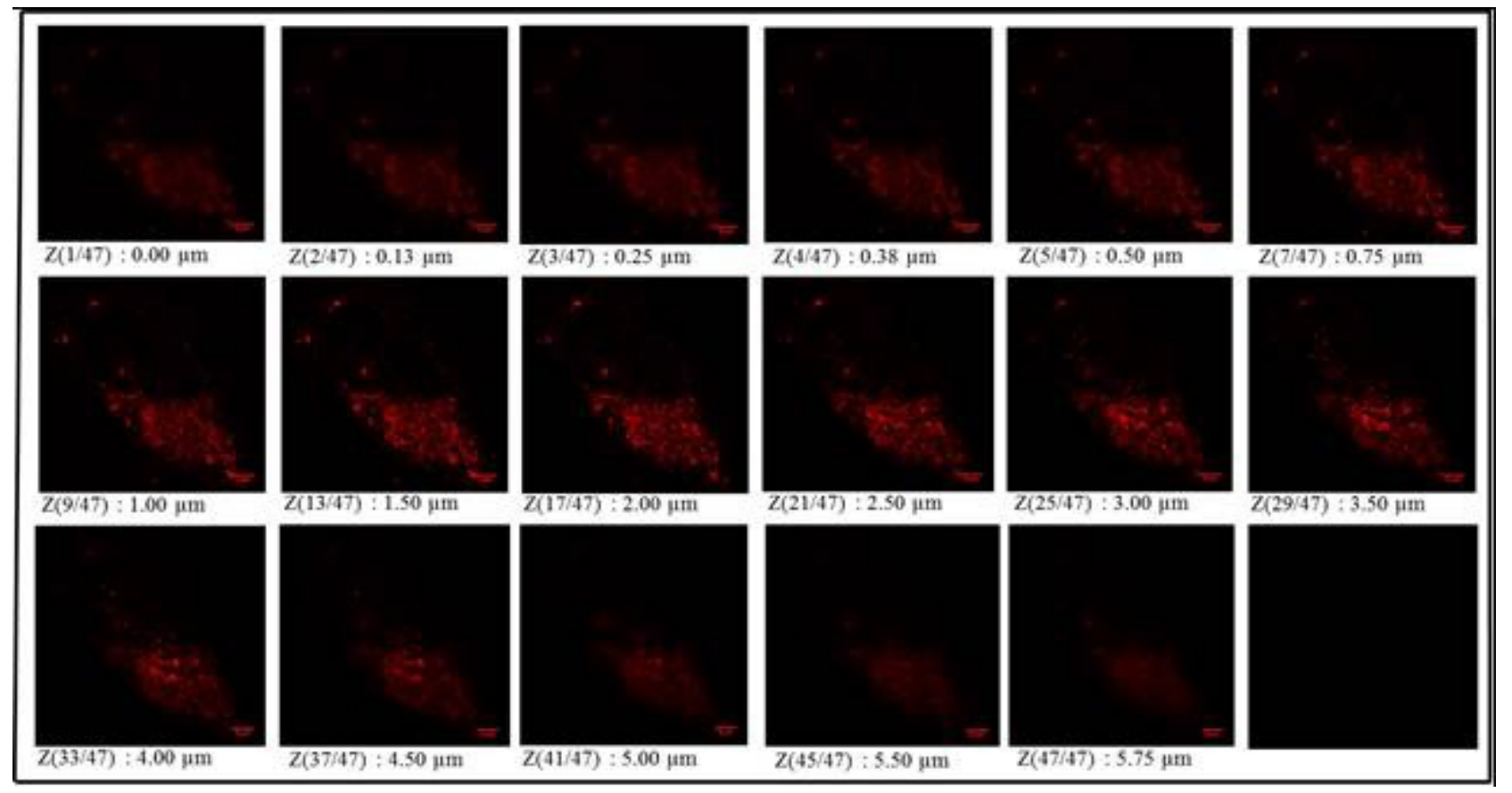

Figure $7 \mathrm{Z}$-Stack through a HeLa cell from the bottom of the cells to the top (left to right) at $0.125 \mu \mathrm{m}$ steps, indicating that CN-PPV loaded SSCL nanoparticles were internalised by HeLa cells post $1 \mathrm{~h}$ incubation. Scale bar $=10 \mu \mathrm{m}$.

As a final study into the uptake of CN-PPV loaded SSCL into cells, three prostate cancer epithelial cell lines were tested. Both, WPE1-NB26 and WPE1-NA22 are derived from RWPE-1, with WPE1-NB26 being a more aggressive prostate cancer line. These show a common lineage that mimics stages in progression from localised malignancy to invasive cancer, and can be used to study carcinogenesis, progression, intervention and chemoprevention ${ }^{38}$. These were incubated with CN-PPV loaded SSCL at a low concentration $(1 \mu \mathrm{g} / \mathrm{ml}$ polymer concentration) for 24 hours. After incubation, the cells were washed with PBS before being fixed in $10 \%$ formalin. As these were plated and fixed in a 24 well plate, the cellular uptake was evaluated at 20x. These cells show less uptake than HeLa cells, which may be because these cells need longer to adhere to the plate. It could therefore not be fully determined whether our particles are suitable with this particular cell line. 


\section{Nanoparticles}
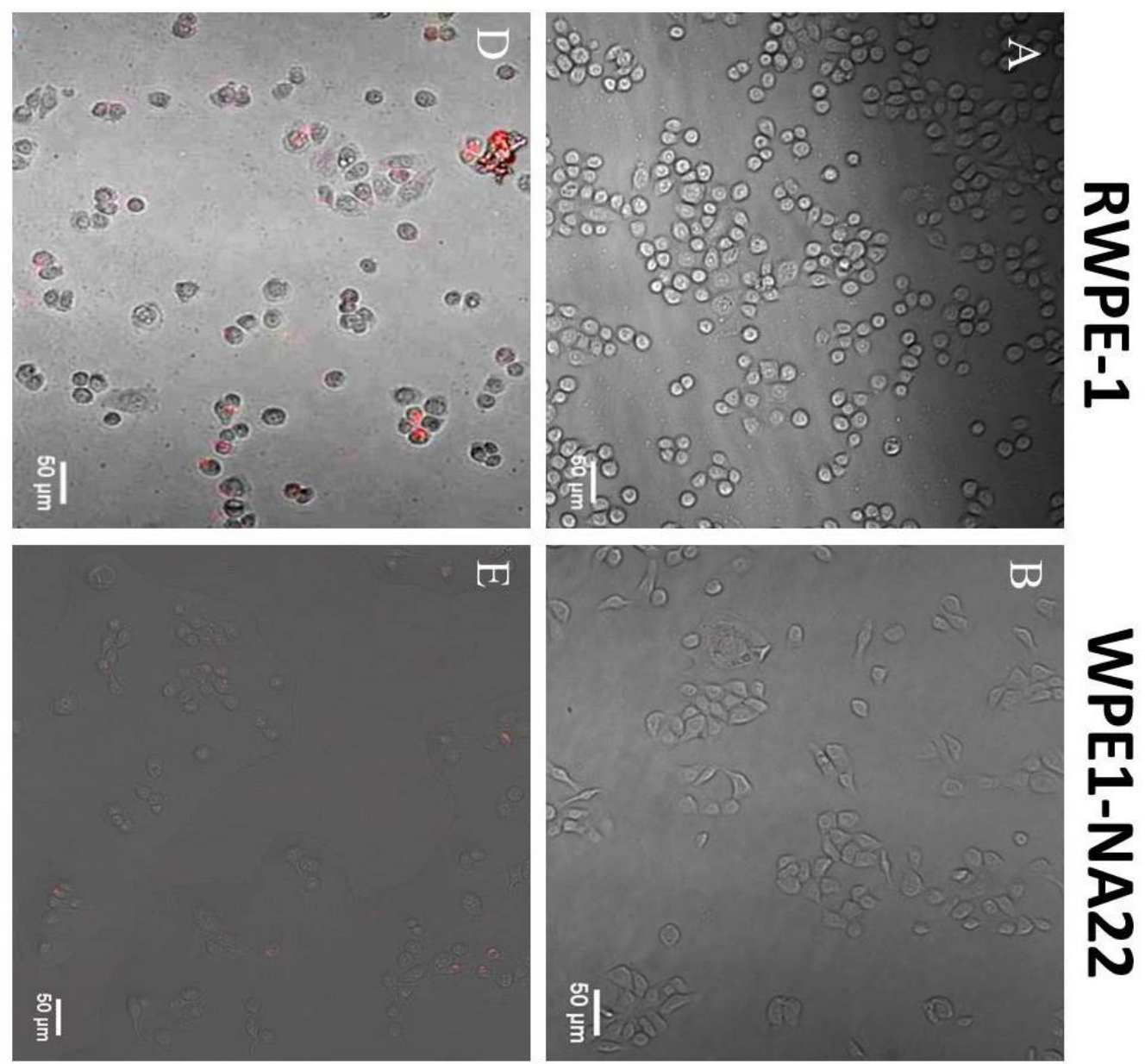

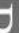
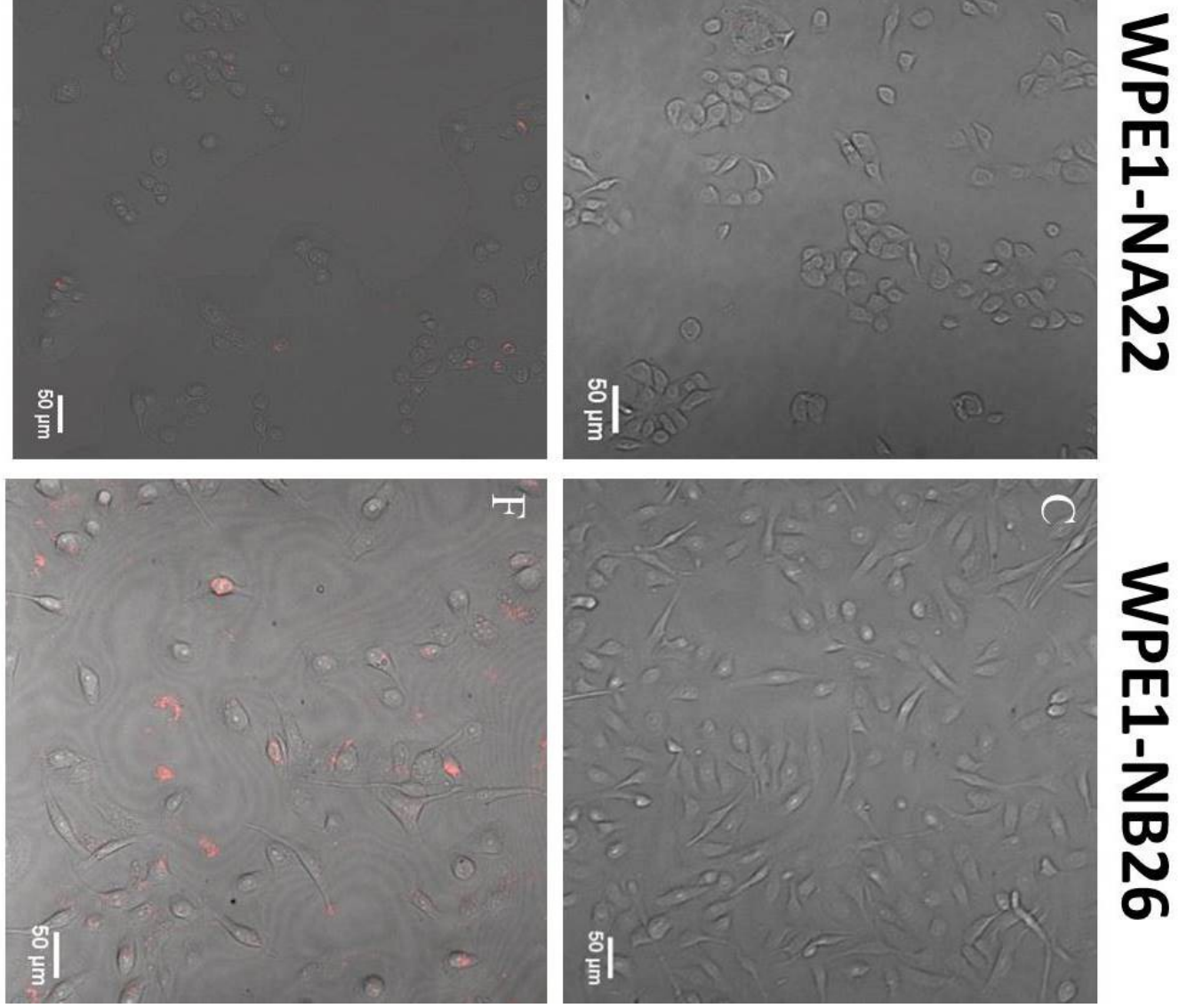

Figure 8 A) RWPE-1 cells untreated or D) treated with CN-PPV loaded SSCL. B) WPE1- NA22 cells untreated or E) treated with CN-PPV loaded SSCL. C) WPE1-NB26 untreated or F) treated with CN-PPV loaded SSCL. Treated cells were incubated with $1 \mu \mathrm{g} / \mathrm{ml}$ of nanoparticles at 20x magnification. Fluorescence was detected in the 570-620 $\mathrm{nm}$ channel. Scale bars are $50 \mu \mathrm{m}$. 


\section{CONCLUSION}

In conclusion, stable, bright, red-emitting fluorescent nanoparticles were successfully synthesised by encapsulating $\mathrm{CN}$ PPV in silica-shell cross-linked polymeric micelles (CP-SSCL) using a silification process. By entrapping the CP within the micelle, and inhibiting the size of the polymeric micelle using a silica shell it was possible to provide small, monodispersed, colloidally stable particles that had high quantum yields and good photo-stability over time. Upon incubation with HeLa cells, bright fluorescence was observed within the cells, particularly in the region surrounding the nuclei of the cells which suggests that uptake occurred. Some particles were uptaken when incubated with the three prostate cancer cell lines, however further work is required with this to ensure optimal uptake. Cytotoxicity studies were carried out using HEK cells, indicating that low nanoparticle concentrations were tolerated, but that high concentrations were lethal. To further this work, conjugation of the pluronic or the silica will need to be undertaken in order to provide target-specific binding and demonstrate usefulness for targeting imaging.

\section{REFERENCES}

[1] Padmanabhan, P., Kumar, A., Kumar, S., Chaudhary, R. K.., Gulyás, B., "Nanoparticles in practice for molecular-imaging applications: An overview," Acta Biomater. 41, 1-16 (2016).

[2] Ferlay, J., Soerjomataram, I., Dikshit, R., Eser, S., Mathers, C., Rebelo, M., Parkin, D. M., Forman, D.., Bray, F., "Cancer incidence and mortality worldwide: Sources, methods and major patterns in GLOBOCAN 2012," Int. J. Cancer 136(5), E359-E386 (2015).

[3] Kochanek, K. D., Xu, J., Murphy, S. L., Minino, A. M.., Kung, H.-C., "National Vital Statistics Reports Deaths : Final Data for 2009," Natl. Cent. Heal. Stat. 60(3), 1-117 (2012).

[4] Jemal, A., Bray, F.., Ferlay, J., "Global Cancer Statistics: 2011," CA Cancer J Clin 49(2), 1,33-64 (1999).

[5] Cancer Research Uk., "Prostate cancer statistics," Prostate cancer Stat., 2016.

[6] Cancer Research Uk., "Bowel cancer statistics," Bowel cancer Stat., 2016.

[7] Li, K., Thomasson, D., Ketai, L., Contag, C., Pomper, M., Wright, M.., Bray, M., "Potential applications of conventional and molecular imaging to biodefense research.," Clin. Infect. Dis. 40(10), 1471-1480 (2005).

[8] Ntziachristos, V., "Going deeper than microscopy: the optical imaging frontier in biology," Nat. Methods 7(8), 603-614, Nature Publishing Group (2010).

[9] Reul, R., Tsapis, N., Hillaireau, H., Sancey, L., Mura, S., Recher, M., Nicolas, J., Coll, J.-L., Fattal, E., et al., "Near infrared labeling of PLGA for in vivo imaging of nanoparticles," Polym. Chem. 3(3), 694 (2012).

[10] Yu, J., Zhang, X., Hao, X., Zhang, X., Zhou, M., Lee, C.-S.., Chen, X., "Near-infrared fluorescence imaging using organic dye nanoparticles," Biomaterials 35(10), 3356-3364 (2014).

[11] Howes, P., Green, M., Levitt, J., Suhling, K.., Hughes, M., "Phospholipid encapsulated semiconducting polymer nanoparticles: Their use in cell imaging and protein attachment," J. Am. Chem. Soc. 132(11), 3989-3996 (2010).

[12] Peng, L., He, M., Chen, B., Wu, Q., Zhang, Z., Pang, D., Zhu, Y.., Hu, B., "Cellular uptake, elimination and toxicity of CdSe/ZnS quantum dots in HepG2 cells," Biomaterials 34(37), 9545-9558, Elsevier Ltd (2013).

[13] Hardman, R., "A Toxicologic Review of Quantum Dots: Toxicity Depends on Physicochemical and Environmental Factors," Environ. Health Perspect. 114(2), 165-172 (2006).

[14] Song, Y., Feng, D., Shi, W., Li, X.., Ma, H., "Parallel comparative studies on the toxic effects of unmodified CdTe quantum dots, gold nanoparticles, and carbon nanodots on live cells as well as green gram sprouts," Talanta 116, 237-244, Elsevier (2013).

[15] Wu, C., Szymanski, C.., McNeill, J., "Preparation and encapsulation of highly fluorescent conjugated polymer nanoparticles," Langmuir 22(7), 2956-2960 (2006).

[16] Geng, J., Liu, J., Liang, J., Shi, H.., Liu, B., “A general approach to prepare conjugated polymer dot embedded silica nanoparticles with a SiO2@CP@SiO2 structure for targeted HER2-positive cellular imaging.," Nanoscale 5(18), 8593-8601 (2013).

[17] Feng, L., Liu, L., Lv, F., Bazan, G. C.., Wang, S., "Preparation and biofunctionalization of multicolor conjugated polymer nanoparticles for imaging and detection of tumor cells," Adv. Mater. 26(23), 3926-3930 (2014).

[18] Wolfbeis, O. S., "An overview of nanoparticles commonly used in fluorescent bioimaging.," Chem. Soc. Rev. 44, 4743-4768, Royal Society of Chemistry (2015).

[19] Pu, K., Chattopadhyay, N.., Rao, J., "Recent advances of semiconducting polymer nanoparticles in in vivo molecular imaging," J. Control. Release 240, 312-322, Elsevier B.V. (2016). 
[20] Zhu, C. L., Liu, L. B., Yang, Q., Lv, F. T.., Wang, S., "Water-Soluble Conjugated Polymers for Imaging, Diagnosis, and Therapy," Chem. Rev. 112(8), 4687-4735 (2012).

[21] Chan, Y.-H.., Wu, P.-J., "Semiconducting Polymer Nanoparticles as Fluorescent Probes for Biological Imaging and Sensing," Part. Part. Syst. Charact. 32(1), 11-28 (2015).

[22] Tuncel, D.., Demir, H. V., "Conjugated polymer nanoparticles," Nanoscale 2(4), 484 (2010).

[23] Pecher, J.., Mecking, S., "Nanoparticles of conjugated polymers," Chem. Rev. 110(10), 6260-6279 (2010).

[24] Zhu, C., Liu, L., Yang, Q., Lv, F.., Wang, S., "Water-soluble conjugated polymers for imaging, diagnosis, and therapy," Chem. Rev. 112(8), 4687-4735 (2012).

[25] Yang, C.-C., Yang, S.-Y., Ho, C.-S., Chang, J.-F., Liu, B.-H.., Huang, K.-W., "Development of antibody functionalized magnetic nanoparticles for the immunoassay of carcinoembryonic antigen: a feasibility study for clinical use.," J. Nanobiotechnology 12(1), 44 (2014).

[26] Peng, H.-S.., Chiu, D. T., "Soft fluorescent nanomaterials for biological and biomedical imaging," Chem. Soc. Rev. 44(14), 4699-4722 (2015).

[27] Vauthier, C.., Bouchemal, K., "Methods for the Preparation and Manufacture of Polymeric Nanoparticles," Pharm. Res. 26(5), 1025-1058 (2009).

[28] Wu, C., Szymanski, C., Cain, Z.., McNeill, J., "Conjugated polymer dots for multiphoton fluorescence imaging," J. Am. Chem. Soc. 129(43), 12904-12905 (2007).

[29] Peters, R., Sandiford, L., Owen, D. M., Kemal, E., Bourke, S., Dailey, L. A.., Green, M., "Red-emitting proteincoated conjugated polymer nanoparticles," Photochem. Photobiol. Sci. 15(11), 1448-1452, The Royal Society of Chemistry (2016).

[30] Ahmad Khanbeigi, R., Abelha, T. F., Woods, A., Rastoin, O., Harvey, R. D., Jones, M.-C., Forbes, B., Green, M. A., Collins, H., et al., "Surface Chemistry of Photoluminescent F8BT Conjugated Polymer Nanoparticles Determines Protein Corona Formation and Internalization by Phagocytic Cells," Biomacromolecules 16(3), 733742 (2015).

[31] Tan, H., Xue, J. M., Shuter, B., Li, X.., Wang, J., "Synthesis of PEOlated Fe3O4@SiO2 nanoparticles via bioinspired silification for magnetic resonance imaging," Adv. Funct. Mater. 20(5), 722-731 (2010).

[32] Tan, H., Zhang, Y., Wang, M., Zhang, Z., Zhang, X., Yong, A. M., Wong, S. Y., Chang, A. Y., Chen, Z.-K., et al., "Silica-shell cross-linked micelles encapsulating fluorescent conjugated polymers for targeted cellular imaging," Biomaterials 33(1), 237-246 (2012).

[33] Tan, H., Liu, N. S., He, B., Wong, S. Y., Chen, Z.-K., Li, X., Wang, J., Yang, J., Lee, J., et al., "Facile synthesis of hybrid silica nanocapsules by interfacial templating condensation and their application in fluorescence imaging," Chem. Commun. 24(41), 6240 (2009).

[34] Xing, L.., Mattice, W. L., "Large internal structures of micelles of triblock copolymers with small insoluble molecules in their cores," Langmuir 14(15), 4074-4080 (1998).

[35] Sun, K., Chen, H., Wang, L., Yin, S., Wang, H., Xu, G., Chen, D., Zhang, X., Wu, C., et al., "Size-dependent property and cell labeling of semiconducting polymer dots," ACS Appl. Mater. Interfaces 6(13), 10802-10812 (2014).

[36] Li, K., Pan, J., Feng, S. S., Wu, A. W., Pu, K. Y., Liu, Y.., Liu, B., "Generic strategy of preparing fluorescent conjugated-polymer-loaded poly(DL-lactide-co-Glycolide) nanoparticles for targeted cell imaging," Adv. Funct. Mater. 19(22), 3535-3542 (2009).

[37] Samuel, I. D. W., Rumbles, G.., Collison, C. J., "Efficient interchain photoluminescence in a high-electronaffinity conjugated polymer," Phys. Rev. B 52(16), R11573-R11576 (1995).

[38] Baker, M. J., Clarke, C., Démoulin, D., Nicholson, J. M., Lyng, F. M., Byrne, H. J., Hart, C. a., Brown, M. D., Clarke, N. W., et al., "An investigation of the RWPE prostate derived family of cell lines using FTIR spectroscopy.," Analyst 135(5), 887-894 (2010). 